\title{
Stark points and the Hida-Rankin p-adic L-function
}

\section{Daniele Casazza \& Victor Rotger}

The Ramanujan Journal

An International Journal Devoted to the Areas of Mathematics Influenced by Ramanujan

\section{ISSN 1382-4090}

\section{Ramanujan J}

DOI 10.1007/s11139-016-9824-y

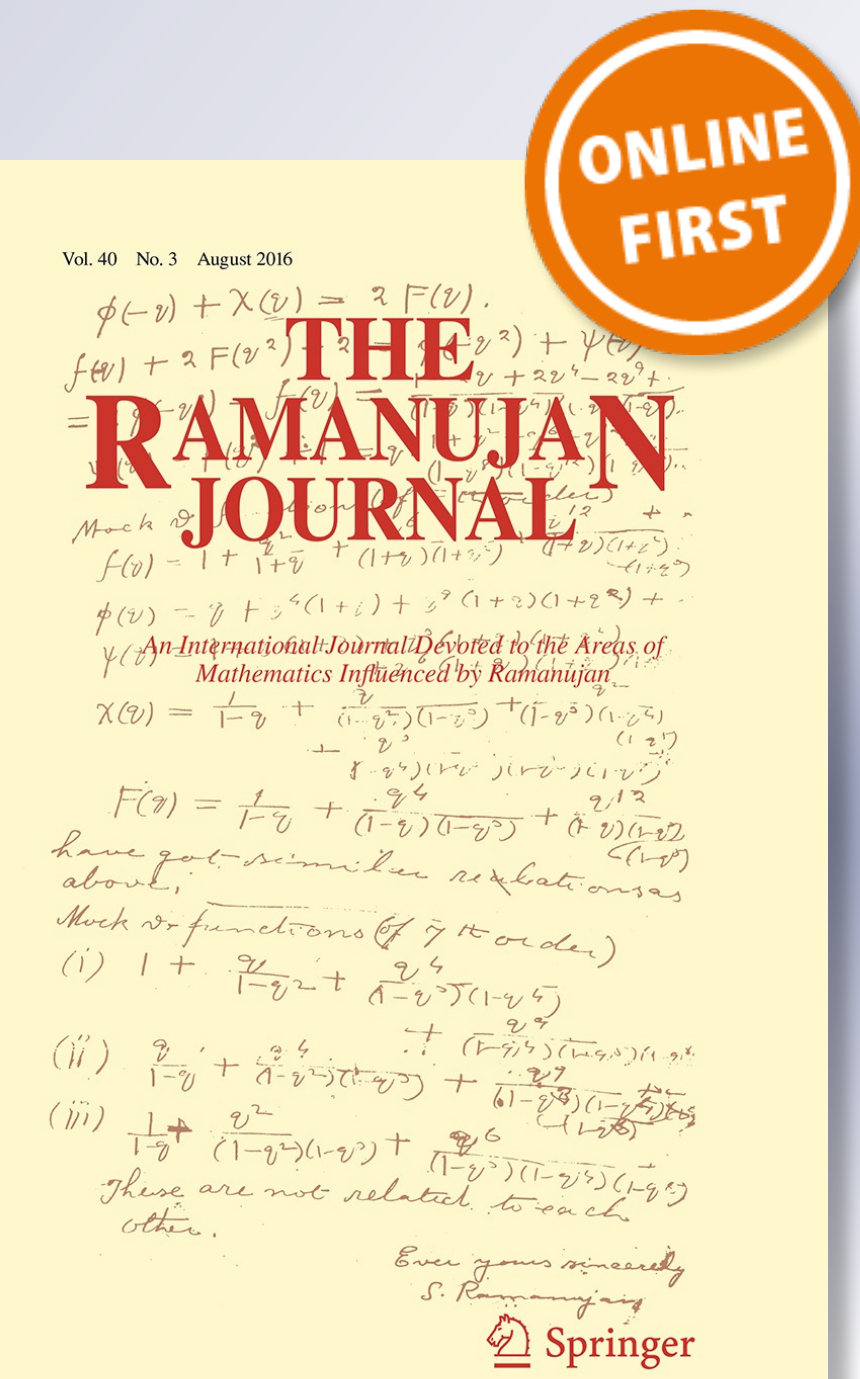

Springer 
Your article is protected by copyright and all rights are held exclusively by Springer Science +Business Media New York. This e-offprint is for personal use only and shall not be selfarchived in electronic repositories. If you wish to self-archive your article, please use the accepted manuscript version for posting on your own website. You may further deposit the accepted manuscript version in any repository, provided it is only made publicly available 12 months after official publication or later and provided acknowledgement is given to the original source of publication and a link is inserted to the published article on Springer's website. The link must be accompanied by the following text: "The final publication is available at link.springer.com". 


\title{
Stark points and the Hida-Rankin $p$-adic $L$-function
}

\author{
Daniele Casazza $^{1,2}$ (1) Victor Rotger ${ }^{3}$
}

Received: 9 May 2016 / Accepted: 16 June 2016

(C) Springer Science+Business Media New York 2016

\begin{abstract}
This article is devoted to the elliptic Stark conjecture formulated by Darmon (Forum Math Pi 3:e8, 2015), which proposes a formula for the transcendental part of a $p$-adic avatar of the leading term at $s=1$ of the Hasse-Weil-Artin $L$-series $L\left(E, \varrho_{1} \otimes \varrho_{2}, s\right)$ of an elliptic curve $E / \mathbb{Q}$ twisted by the tensor product $\varrho_{1} \otimes \varrho_{2}$ of two odd 2-dimensional Artin representations, when the order of vanishing is two. The main ingredient of this formula is a $2 \times 2 p$-adic regulator involving the $p$-adic formal group logarithm of suitable Stark points on $E$. This conjecture was proved by Darmon (Forum Math Pi 3:e8, 2015) in the setting where $\varrho_{1}$ and $\varrho_{2}$ are induced from characters of the same imaginary quadratic field $K$. In this note, we prove a refinement of this result that was discovered experimentally by Darmon (Forum Math Pi 3:e8, 2015, [Remark 3.4]) in a few examples. Namely, we are able to determine the algebraic constant up to which the main theorem of Darmon (Forum Math Pi 3:e8, 2015) holds in a particular setting where the Hida-Rankin $p$-adic $L$-function associated to a pair of Hida families can be exploited to provide an alternative proof of the same result. This constant encodes local and global invariants of both $E$ and $K$.
\end{abstract}

Keywords Elliptic curves · Birch and Swinnerton-Dyer conjecture - Special values · p-Adic modular forms · Elliptic units

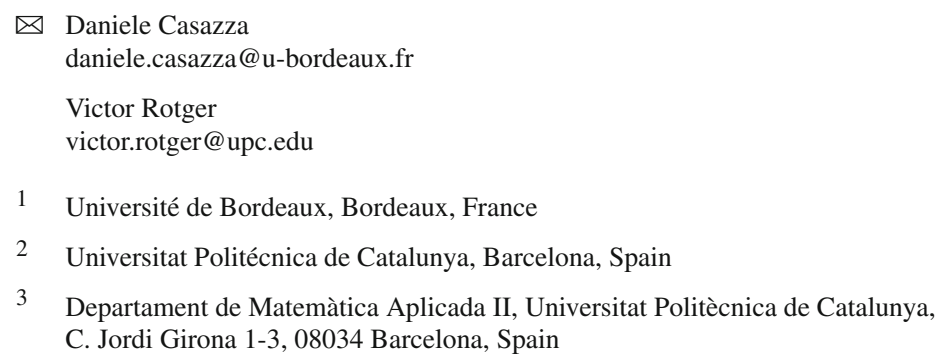


Mathematics Subject Classification 11G05 - 11G40 - 11F67 · 11F33 - $11 \mathrm{G} 16$

\section{Introduction}

Let $E / \mathbb{Q}$ be an elliptic curve of conductor $N_{E}$ and let $f \in S_{2}\left(N_{E}\right)$ denote the eigenform associated to it by modularity. Let in addition

$$
\varrho: G_{\mathbb{Q}} \longrightarrow \mathbf{G L}\left(V_{\varrho}\right)=\mathbf{G L}_{n}(L)
$$

be an Artin representation with values in a finite extension $L / \mathbb{Q}$ and factoring through the Galois group $\mathrm{Gal}(H / \mathbb{Q})$ of a finite Galois extension $H / \mathbb{Q}$. Here $V_{\varrho}$ is the $L\left[G_{\mathbb{Q}}\right]$ module underlying the Galois representation $\varrho$.

We define the $\varrho$-isotypical component of the Mordell-Weil group of $E(H)$ as

$$
E(H)^{\varrho}=\operatorname{Hom}_{\mathrm{Gal}(H / \mathbb{Q})}\left(V_{\varrho}, E(H) \otimes L\right),
$$

and set

$$
r(E, \varrho):=\operatorname{dim}_{L} E(H)^{\varrho} .
$$

Let $L(E, \varrho, s)$ denote the Hasse-Weil-Artin $L$-series of the twist of $E$ by $\varrho$. This $L$-function is expected to admit analytic continuation to the whole complex plane and to satisfy a functional equation relating the values at $s$ and $2-s$, although this is only known in a few cases, including the ones we consider in this note. Assuming these properties, we may define the analytic rank of the pair $(E, \varrho)$ as

$$
r_{\mathrm{an}}(E, \varrho):=\operatorname{ord}_{s=1} L(E, \varrho, s)
$$

The equivariant refinement of the Birch and Swinnerton-Dyer conjecture (cf. [15, 32]) for the twist of $E$ by $\varrho$ predicts that

$$
r(E, \varrho) \stackrel{?}{=} r_{\mathrm{an}}(E, \varrho) .
$$

The equality (1) is known to be true in rather few cases, always under the assumption that $r_{\text {an }}(E, \varrho)=0$ or $r_{\text {an }}(E, \varrho)=1$. We refer to [6,13] for the latest developments in this direction. In particular, one is often at a loss to construct nontrivial points in $E(H)^{\varrho}$.

In the recent work [10], Darmon, Lauder, and Rotger propose a new approach for computing (linear combinations of logarithms of) nonzero elements in $E(H)^{\varrho}$ for a wide class of Artin representations $\varrho$ under the assumption that $r_{\text {an }}(E, \varrho) \leq 2$. More precisely, $\varrho$ is allowed to be any irreducible constituent of the tensor product

$$
\varrho=\varrho_{1} \otimes \varrho_{2}
$$


of any pair of odd, two-dimensional Galois representations $\varrho_{1}$ and $\varrho_{2}$ of conductors $N_{1}$ and $N_{2}$, respectively, satisfying

$$
\left(N_{E}, N_{1} N_{2}\right)=1, \quad \operatorname{det}\left(\varrho_{2}\right)=\operatorname{det}\left(\varrho_{1}\right)^{-1} \text { and } r_{\text {an }}\left(E, \varrho_{1} \otimes \varrho_{2}\right)=2 \text {. }
$$

After the ground-breaking works of Buzzard-Taylor, Khare-Wintenberger, and others, we now know that these representations are modular and hence $\varrho_{1}$ and $\varrho_{2}$ are isomorphic to the Deligne-Serre Artin representation associated to eigenforms

$$
g \in M_{1}(N, \chi), \quad h \in M_{1}\left(N, \chi^{-1}\right),
$$

respectively, where the level $N$ might be taken to be the least common multiple of $N_{E}, N_{1}$, and $N_{2}$, and the nebentype $\chi=\operatorname{det}\left(\varrho_{1}\right)$ is the determinant of $\varrho_{1}$ regarded as a Dirichlet character.

Fix a prime $p \nmid N$ at which $g$ satisfies the classicality hypotheses $\mathrm{C}-\mathrm{C}^{\prime}$ introduced in [10]. The main conjecture of [10] is a formula relating

- a $p$-adic iterated integral associated to the triple $(f, g, h)$ of eigenforms,

- an explicit linear combination of formal group logarithms of points of infinite order on $E(H)$, and

- the $p$-adic logarithm of a Gross-Stark unit associated to the adjoint of $g$.

As shown in [10, Sect. 2], the $p$-adic iterated integral can be recast as the value of the triple-product Harris-Tilouine $p$-adic $L$-function constructed in [12, Sect. 4.2] at a point of weights $(2,1,1)$. In this paper, we place ourselves in a setting where $h$ is Eisenstein and Harris-Tilouine's $p$-adic $L$-function alluded to above can be replaced with the more standard Rankin $p$-adic $L$-function of Hida [20, Sect. 7.4]. This observation is crucial for our purposes, for the latter $p$-adic $L$-function is more amenable to explicit computations.

The linear combination of logarithms of points mentioned above arises as the determinant of a $2 \times 2$ matrix introduced in [10] that plays the role of a $p$-adic avatar of the regulator in the classical setting. Note however that this $p$-adic regulator does not coincide with the one considered by Mazur et al. [28], as the $p$-adic height function is replaced in [10] with the formal logarithm on the elliptic curve.

In some instances, as we shall see below, the logarithm of one of the points may be isolated in such a way that the conjectural expression suggested in [10, ConjectureES] gives rise to a formula that allows to compute the point in terms of more accessible quantities which include the $p$-adic iterated integral and logarithms of global units and points that are rational over smaller number fields.

To describe more precisely our main result, let $K$ be an imaginary quadratic field of discriminant $-D_{K}$ with $D_{K} \geq 7$ and let $\mathcal{O}_{K}$ denote its ring of integers. Let also $h_{K}=\left|\mathrm{Cl}_{K}\right|$ denote the class number, $g_{K}=\left[\mathrm{Cl}_{K}: \mathrm{Cl}_{K}^{2}\right]$ be the number of genera, and $\chi_{K}$ be the quadratic Dirichlet character associated to $K / \mathbb{Q}$.

Let $c \geq 1$ be a fixed positive integer relatively prime to $N_{E}$ and let $H / K$ denote the ring class field of $K$ of conductor $c$. Let

$$
\psi: \operatorname{Gal}(H / K) \longrightarrow \overline{\mathbb{Q}}^{\times}
$$


be a character of finite order and

$$
g:=\theta(\psi) \in M_{1}\left(D_{K} c^{2}, \chi_{K}\right)
$$

be the theta series associated to $\psi$. This is an eigenform of level $D_{K} c^{2}$ and nebentype character $\chi_{K}$. The form $g$ is cuspidal if and only if $\psi^{2} \neq 1$.

Let $\mathbb{Q}_{\psi}$ denote the finite extension generated by the values of $\psi$. Let $\varrho_{\psi}=\operatorname{Ind}_{\mathbb{Q}}^{K}(\psi)$ denote the odd two-dimensional Artin representation of $G_{\mathbb{Q}}$ induced by $\psi$ and write $V_{\psi}$ for the underlying two-dimensional $\mathbb{Q}_{\psi}$-vector space.

Fix now an odd rational prime $p=\wp \bar{\gamma} \nmid N_{E} D_{K} c^{2}$ that splits in $K$. The choice of the ideal $\wp$ above $p$ determines a Frobenius element $\operatorname{Fr}_{p}$ in $\operatorname{Gal}(H / K)$. The eigenvalues of $\operatorname{Fr}_{p}$ acting on $V_{\psi}$ are

$$
\alpha=\psi\left(\operatorname{Fr}_{p}\right), \quad \beta=\bar{\psi}\left(\operatorname{Fr}_{p}\right)=\alpha^{-1}
$$

As mentioned above, we assume that $g$ satisfies the classicality hypotheses $\mathrm{C}-\mathrm{C}^{\prime}$ of [10] at the prime $p$. When $g$ is cuspidal, i.e., $\psi^{2} \neq 1$, one can verify ${ }^{1}$ that in our setting the hypothesis is equivalent to assuming that $\alpha \neq \beta$. When $\theta(\psi)$ is Eisenstein, i.e., $\psi^{2}=1$, one expects ${ }^{2}$ that hypotheses $C-C^{\prime}$ are always satisfied in our case.

Remark 1.1 This assumption might appear unmotivated when one encounters it for the first time. In the case where $g$ is cuspidal, a striking recent result of Bellaïche and Dimitrov [2] shows that hypotheses $\mathrm{C}-\mathrm{C}^{\prime}$ ensure that the points in the eigencurve associated to either of the two ordinary $p$-stabilizations $g_{\alpha}, g_{\beta}$ of $g$ are smooth and the weight map is étale at these points.

Building on their work, it was further shown in [10, Sect. 1] that this assumption implies that all overconvergent generalized eigenforms with the same system of Hecke eigenvalues as $g_{\alpha}$ are necessarily classical. This in turn guarantees that the $p$-adic iterated integral we will consider in (5) below is well defined.

Finally, it is also worth mentioning that hypotheses $\mathrm{C}-\mathrm{C}^{\prime}$ imply that the elliptic unit $u_{\psi^{2}}$ introduced in (2) below may be characterized, up to multiplication by scalars in $\mathbb{Q}_{\psi}^{\times}$, as follows: up to $\mathbb{Q}_{\psi}^{\times}$, there is a single $\mathrm{Gal}(H / K)$-equivariant homomorphism $\varphi: \mathbb{Q}_{\psi}\left(\psi^{2}\right) \longrightarrow \mathcal{O}_{H}^{\times} \otimes \mathbb{Q}_{\psi}$ and $u_{\psi^{2}} \operatorname{spans} \operatorname{Im}(\varphi)$.

The choice of $\wp$ determines an embedding $K \hookrightarrow \mathbb{Q}_{p}$ that we extend to an embedding $H \hookrightarrow \mathbb{C}_{p}$. Write $H_{p}$ for the completion of $H$ with respect to this embedding.

\footnotetext{
1 Indeed, when $g$ is cuspidal this hypothesis not only asks that $\alpha \neq \beta$ but also that there should exist no real quadratic field $F$ in which $p$ splits such that $\varrho_{g} \simeq \operatorname{Ind}_{F}^{\mathbb{Q}}(\xi)$ for some character $\xi$ of $F$. However, in our CM setting, the existence of a character $\xi$ of a real quadratic field $F$ such that $\operatorname{Ind}_{\mathbb{Q}}^{K}(\psi) \simeq \operatorname{Ind}_{\mathbb{Q}}^{F}(\xi)$ implies that $\mathrm{Gal}(H / K) \simeq C_{4}$. Then $F$ is the single real quadratic field contained in the quadratic extension of $K$ cut out by $\psi^{2}$, and the condition $\alpha \neq \beta$ implies that $p$ cannot split in $F$.

2 Indeed, when $\theta(\psi)$ is Eisenstein, it is shown in [10, Sect. 1] that a necessary condition for hypotheses $\mathrm{C}-\mathrm{C}^{\prime}$ to hold is that $\alpha=\beta$, but this is automatically satisfied because $\psi^{2}=1$. As explained in loc. cit., this is also expected to be a sufficient condition.
} 
Note that there is a natural isomorphism of Gal $(H / K)$-modules

$$
E(H)^{\varrho \psi}=E(H)^{\psi} \oplus E(H)^{\bar{\psi}}
$$

where

$$
E(H)^{\psi}=\left\{P \in E(H) \otimes \mathbb{Q}_{\psi}: P^{\sigma}=\psi(\sigma) P \text { for all } \sigma \in \operatorname{Gal}(H / K)\right\}
$$

and $E(H)^{\bar{\psi}}$ is defined likewise.

Throughout, we impose the classical Heegner hypothesis.

Assumption 1.2 There exists an integral ideal $\mathfrak{N}$ in $\mathcal{O}_{K}$ such that $\mathcal{O}_{K} / \mathfrak{N} \simeq \mathbb{Z} / N_{E} \mathbb{Z}$.

This assumption guarantees the existence of nontrivial elliptic units in $H^{\times}$and Heegner points in $E(H)$ arising from the classical modular curve $X_{0}\left(N_{E}\right)$. In particular, there exists a canonical unit (cf. e.g., [9, Sect. 1], [31, pp. 15-16]) associated to the character $\psi^{2}$ defined as

$$
u_{\psi^{2}}= \begin{cases}\operatorname{any} p-\operatorname{unitin} \mathcal{O}_{H}\left[\frac{1}{p}\right]^{\times} \operatorname{satisfying}\left(u_{\wp}\right)=\wp^{h_{K}}, & \text { if } \quad \psi^{2}=1 \\ \sum_{\sigma \in \operatorname{Gal}(H / K)} \psi^{2}(\sigma) u^{\sigma} \in \mathcal{O}_{H}^{\times} \otimes \mathbb{Q}_{\psi}, & \text { if } \quad \psi^{2} \neq 1\end{cases}
$$

Likewise, associated to $\psi$ there is the canonical point (cf.e.g., [18, I. Sect. 6])

$$
P_{\psi}:=\sum_{\sigma \in \operatorname{Gal}(H / K)} \psi(\sigma) x^{\sigma} \in E(H)^{\psi^{-1}} \subset E(H)^{V_{\psi}}
$$

In (2) and (3), $u$ and $x$ are arbitrary choices of an elliptic unit and Heegner point with $\mathrm{CM}$ by $\mathcal{O}_{K}$, respectively. Since, up to sign, all such choices lie in the same orbit under Gal $(H / K), u_{\psi^{2}}$ and $P_{\psi}$ do not depend on this, again up to sign. Notice that the convention is different to the one usually taken in literature in the sense that $\sigma$ acts on $P_{\psi}$ as $\psi(\sigma)^{-1}$ instead of $\psi(\sigma)$.

Set $N=\operatorname{lcm}\left(D_{K} c^{2}, N_{E}\right)$. Write $S_{2}(N)[f]$ (resp. $M_{1}\left(N, \chi_{K}\right)[g]$ ) for the subspace of $S_{2}(N)$ (resp. of $M_{1}\left(N, \chi_{K}\right)$ ) consisting of modular forms that are eigenvectors for all good Hecke operators $T_{\ell}, \ell \nmid N$, with the same eigenvalues as $f$ (resp. $g$ ).

Fix a modular form $\breve{f}=\sum_{n \geq 1} a_{n}(\breve{f}) q^{n} \in S_{2}(N)[f]$ and let

$$
\breve{f}^{[p]}=\sum_{p \nmid n} a_{n}(\breve{f}) q^{n}
$$

denote the $p$-depletion of $\breve{f}$.

Fix as well a modular form $\breve{g} \in M_{1}\left(N, \chi_{K}\right)[g]$ and let

$$
\breve{g}_{\alpha}(q)=\breve{g}(q)-\beta_{g} \breve{g}\left(q^{p}\right) \in M_{1}\left(p N, \chi_{K}\right)
$$

denote the ordinary stabilization of $\breve{g}$ on which $U_{p}$ acts with eigenvalue $\alpha$. 
Associated to the pair $\left(\breve{f}, \breve{g}_{\alpha}\right)$, we may define a $p$-adic iterated integral as follows. Let $h:=\operatorname{Eis}_{1}\left(1, \chi_{K}\right) \in M_{1}\left(D_{K}, \chi_{K}\right)$ be the Eisenstein series associated to the Dirichlet character $\chi_{K}$, as defined, e.g., in [5, Sect. 2.1.2]. Let also

$$
\breve{h}:=E_{1, \chi_{K, N}} \in M_{1}\left(N, \chi_{K}\right)[h]
$$

denote the Eisenstein series of level $N$ in the isotypic eigenspace of $h$ that we introduce in (15) below. Let

$$
e_{\mathrm{ord}}: M_{1}^{\mathrm{oc}}\left(N, \chi_{K}\right) \longrightarrow M_{1}^{\mathrm{oc}, \text { ord }}\left(N, \chi_{K}\right)
$$

denote Hida's ordinary idempotent on the space of overconvergent modular forms of weight 1 , tame level $N$, and tame character $\chi_{K}$. Let

$$
e_{g_{\alpha}}^{*}: M_{1}^{\mathrm{oc}, \text { ord }}\left(N, \chi_{K}\right) \longrightarrow M_{1}^{\mathrm{oc}, \text { ord }}\left(N, \chi_{K}\right)\left[\left[g_{\alpha}^{*}\right]\right]
$$

denote the projection onto the generalized eigenspace attached to $g_{\alpha}^{*}$.

Letting $d=q \frac{d}{d q}$ denote Serre's $p$-adic derivative operator, the overconvergent ordinary modular form

$$
e_{g_{\alpha}}^{*} e_{\mathrm{ord}}\left(d^{-1} \breve{f}^{[p]} \times E_{1, \chi_{\mathrm{K}, \mathrm{N}}}\right)
$$

lies in the space of classical modular forms $M_{1}\left(p N, \chi_{K}\right)\left[g_{\alpha}^{*}\right]$ of weight 1 , level $p N$, and nebentype $\chi_{K}$, with coefficients in $\mathbb{Q}_{p}$, as explained in Remark 1.1.

Let $\breve{\gamma}_{g_{\alpha}}$ be an element in the $\mathbb{Q}_{\psi}$-dual space of $M_{1}\left(p N, \chi_{K}\right)\left[g_{\alpha}^{*}\right]$; more specifically, we take it to be the one associated to $\breve{g}_{\alpha}$ in [10, Proposition 2.6]. By extending scalars, we may regard $\breve{\gamma}_{g_{\alpha}}$ as a $\mathbb{Q}_{p}(\psi)$-linear functional on $M_{1}\left(p N, \chi_{K}\right)\left[g_{\alpha}^{*}\right]$ as well.

Following [10], define

$$
\int_{\breve{\gamma}_{g_{\alpha}}} \breve{f} \cdot E_{1, \chi_{\mathrm{K}, \mathrm{N}}}:=\breve{\gamma}_{g_{\alpha}}\left(e_{g_{\alpha}}^{*} e_{\mathrm{ord}}\left(d^{-1} \breve{f}^{[p]} \times E_{1, \chi_{\mathrm{K}, \mathrm{N}}}\right)\right) \in \mathbb{C}_{p} .
$$

In [10, Theorem 3.3], it was proved a statement that, specialized to our setting, asserts the following. Let $\log _{p}$ denote the usual branch of the $p$-adic logarithm on $H_{p}^{\times}$ that satisfies $\log _{p}(p)=0$ and let $\log _{E, p}$ denote the formal group logarithm on $E / H_{p}$.

Theorem 1.3 Assume $r_{\mathrm{an}}(E / K, \psi)=1$. There exists a finite extension $L$ of $\mathbb{Q}_{\psi}$ and a scalar $\lambda(\breve{f}, \breve{g}) \in L$ such that

$$
\int_{\breve{\gamma}_{g \alpha}} \breve{f} \cdot E_{1, \chi_{\mathrm{K}, \mathrm{N}}}=\lambda(\breve{f}, \breve{g}) \cdot \frac{\log _{E, p}^{2}\left(P_{\psi}\right)}{\log _{p}\left(u_{\psi^{2}}\right)} .
$$

Moreover, there is a suitable choice of $\breve{f}$ and $\breve{g}$ such that $\lambda(\breve{f}, \breve{g}) \neq 0$.

Several questions arise naturally in the light of the above statement: 
- Can the field $L$ be determined?

- Are there explicit choices of $\breve{f}$ and $\breve{g}$ for which $\lambda(\breve{f}, \breve{g}) \neq 0$ ?

- Can the scalar $\lambda(\breve{f}, \breve{g})$ be computed explicitly?

- Does $\lambda(\breve{f}, \breve{g})$ have any arithmetical meaning?

The aim of this note is answering these questions by proving an explicit formula for the scalar $\lambda(\breve{f}, \breve{g})$ in terms of local and global arithmetic invariants of $E$ and $\psi$. In doing this, we prove as a particular case a formula that was already conjectured and verified numerically in [10, Remark 3.4 and (45)].

While the main conjecture of [10] may be regarded as a $p$-adic analogue of the rank part of the classical equivariant Birch and Swinnerton-Dyer conjecture, our main result provides a precise formula for the leading term in the particular setting we have placed ourselves. Hence, Theorem 1.4 below may be regarded as a $p$-adic avatar of the formula for the leading term predicted by the conjecture of Birch and Swinnerton-Dyer, and we hope it may suggest a $p$-adic variant of the classical equivariant Tamagawa number conjecture; more details on this may appear elsewhere.

The reader may also find Theorem 1.4 interesting from the computational point of view, as it provides an explicit $p$-adic formula for the Heegner point $P_{\psi}$ in $E\left(H_{p}\right) / E\left(H_{p}\right)_{\text {tors. }}$. Namely,

$$
P_{\psi}=\exp _{E, p}\left(\sqrt{\frac{\log _{p}\left(u_{\psi^{2}}\right)}{\lambda(\breve{f}, \breve{g})} \cdot \int_{\breve{\gamma}_{g \alpha}} \breve{f} \cdot E_{1, \chi_{K, N}}}\right) .
$$

Let $\mathbb{Q}\left(f_{N}\right)$ denote the finite extension of $\mathbb{Q}$ generated by the roots of the Hecke polynomials $T^{2}-a_{q}(f) T+q$ for all primes $q \mid N, q \nmid N_{E}$. Note that if $\breve{f} \in S_{2}(N)[f]$ is chosen to be a normalized eigenvector for all good and bad Hecke operators $T_{\ell}$ for all primes $\ell$, then the fourier coefficients of $\breve{f}$ lie in $\mathbb{Q}\left(f_{N}\right)$. In a similar way, observe also that if $\breve{g} \in M_{1}(N)[g]$ is chosen to be an eigenvector for all good and bad Hecke operators, then the fourier coefficients of $\breve{g}$ lie in $\mathbb{Q}_{\psi}$. Write $\mathbb{Q}_{\psi}\left(f_{N}\right)$ for the compositum of $\mathbb{Q}\left(f_{N}\right)$ and $\mathbb{Q}_{\psi}$.

Theorem 1.4 (i) If $\breve{f}$ and $\breve{g}$ are chosen to be eigenvectors for all good and bad Hecke operators, then $L$ can be taken to be $\mathbb{Q}_{\psi}\left(f_{N}\right)$ and $\lambda(\breve{f}, \breve{g}) \neq 0$.

(ii) Assume that $D_{K}=N_{E}$ and $c=1$. Then the following formula holds true for $\breve{f}=f, \breve{g}=g$ :

$$
\lambda(f, g)=\frac{\left(p-a_{p}(f) \psi(\bar{\wp})+\psi^{2}(\bar{\wp})\right)^{2}}{p} \cdot \frac{\lambda_{0}}{h_{K} g_{K}},
$$

where

$$
\lambda_{0}=\left\{\begin{array}{lll}
\frac{1}{p-1} & \text { if } \psi^{2}=1, & \text { that is to say, if } g \text { is Eisenstein } \\
\frac{12}{p-(p+1) \psi^{-2}(\bar{\varnothing})+\psi^{-4}(\bar{\wp})} & \text { if } \quad \psi^{2} \neq 1, & \text { that is to say, if } g \text { is cuspidal. }
\end{array}\right.
$$


Note that in the special case in which $N_{E}$ is prime and $\psi=1$, we obtain

$$
\lambda(f, g)=\frac{\left|E\left(\mathbb{F}_{p}\right)\right|^{2}}{p(p-1) h_{K}} .
$$

As reported in [10, Remark 3.4 and (45)], this formula was verified numerically in several examples, and here it is proved unconditionally.

The proof of Theorem 1.4 actually provides an alternative proof of Theorem 1.3 in the setting considered here. As in loc.cit., we compare the values of several $p$-adic $L$-functions at several points lying outside the region of interpolation, the main novelty with respect to [10] being that we exploit Hida-Rankin $p$-adic $L$-function associated to the convolution of two Hida families, instead of the triple-product Harris-Tilouine $p$-adic $L$-function associated to a triple of Hida families. Since the former has been extensively studied in the literature, this alternative approach allows us to perform the explicit computations that are needed in order to derive the sought-after refined formula.

\section{Hecke characters, theta series, and Katz's $p$-adic $L$-function}

Let $K / \mathbb{Q}$ be an imaginary quadratic field of discriminant $-D_{K}$ and let $\mathfrak{c} \subset \mathcal{O}_{K}$ be an integral ideal. Let $I_{\mathfrak{c}}$ denote the group of fractional ideals of $K$ that are coprime to $c$.

A Hecke character of infinity type $\left(\kappa_{1}, \kappa_{2}\right)$ of $K$ is a homomorphism

$$
\psi: I_{\mathfrak{c}} \longrightarrow \mathbb{C}^{\times}
$$

such that

$$
\psi((\alpha))=\alpha^{\kappa_{1}} \bar{\alpha}^{\kappa_{2}}
$$

for all $\alpha \equiv 1(\bmod \mathfrak{c})$. The conductor of $\psi$ is the largest ideal $\mathfrak{c}_{\psi}$ for which this holds. Let us introduce some basic notations and terminology:

- The norm map $\mathbf{N}_{K}:=\left|N_{\mathbb{Q}}^{K}\right|: I_{\mathfrak{c}} \longrightarrow \mathbb{C}^{\times}$gives rise to a Hecke character of infinity type $(1,1)$ and conductor 1 .

- For any Hecke character $\psi$ of infinity type $\left(\kappa_{1}, \kappa_{2}\right)$ define $\psi^{\prime}(\mathfrak{a})=\psi(\overline{\mathfrak{a}})$, where $\bar{x}$ denotes complex conjugation; we say that $\psi$ is self-dual, or anticyclotomic, if $\psi \psi^{\prime}=\mathbf{N}_{K}^{\kappa_{1}+\kappa_{2}}$.

- A Hecke character of finite order (or infinity type $(0,0)$ ) can be regarded as a character of $G_{K}=\mathrm{Gal}(\bar{K} / K)$ via class field theory. We continue to denote by $\psi$ the resulting character, which is anticyclotomic.

- The central character $\varepsilon_{\psi}$ of $\psi$ is the single Dirichlet character satisfying

$$
\left.\psi\right|_{\mathbb{Q}}=\varepsilon_{\psi} \mathbf{N}_{K}^{\kappa_{1}+\kappa_{2}}
$$

The following lemma is well known. 
Lemma 2.1 Let $\psi$ be a Hecke character of finite order. The following are equivalent:

(1) $\psi$ is a ring class character.

(2) $\operatorname{Ind}_{\mathbb{Q}}^{K}(\psi)$ is a self-dual representation.

(3) The central character of $\psi$ is trivial.

Given a Hecke character of $K$ of infinity type $\left(\kappa_{1}, \kappa_{2}\right)$, we can associate to it a theta series as follows. Define the quantities

$$
a_{n}(\psi)=\sum_{\mathfrak{a} \in I_{\mathfrak{c} \psi}^{n}} \psi(\mathfrak{a})
$$

where $I_{\mathfrak{c}_{\psi}}^{n}$ is the set of the ideals in $I_{\mathfrak{c}_{\psi}}$ whose norm is $n$. Define also $a_{0}(1)=h_{K} / w_{K}$ and $a_{0}(\psi)=0$ otherwise. As shown in [21],

$$
\theta_{\psi}:=\sum_{n \geq 0} a_{n}(\psi) q^{n}=\sum_{n \geq 0} a_{n}\left(\theta_{\psi}\right) q^{n} \in M_{\kappa_{1}+\kappa_{2}}\left(D_{K} N_{\mathbb{Q}}^{K}\left(\mathfrak{c}_{\psi}\right), \chi_{K} \varepsilon_{\psi}\right)
$$

is the $q$-expansion of a normalized newform of weight $\kappa_{1}+\kappa_{2}$, level $D_{K} N_{\mathbb{Q}}^{K}\left(\mathfrak{c}_{\psi}\right)$, and nebentype $\chi_{K} \varepsilon_{\psi}$. Moreover $\theta_{\psi}$ is Eisenstein if and only if $\psi=\psi^{\prime}$; otherwise $\theta_{\psi}$ is a cusp form.

Associated to $\psi$ and $\theta_{\psi}$, there are the Hecke $L$-functions

$$
L(\psi, s):=\prod_{\mathfrak{p}}\left(1-\frac{\psi(\mathfrak{p})}{N_{\mathbb{Q}}^{K} \mathfrak{p}^{s}}\right)^{-1} \text { and } L\left(\theta_{\psi}, s\right):=\sum_{n \geq 1} \frac{a_{n}\left(\theta_{\psi}\right)}{n^{s}} .
$$

They can be extended to meromorphic functions on $\mathbb{C}$. Since they coincide in a common region of convergence, they are actually the same function. From the definitions, it is easy to verify that for any $k \in \mathbb{Z}$ we have

$$
L(\psi, s)=L\left(\psi \mathbf{N}_{K}^{k}, s+k\right)
$$

\subsection{Katz's two-variable $p$-adic $L$-function}

Assume $D_{K} \geq 7$ and let $\mathfrak{c} \subseteq \mathcal{O}_{K}$ be an integral ideal. Fix a prime $p=\wp \bar{\varnothing}$ that splits in $K$.

Denote by $\Sigma$ the set of Hecke characters of $K$ of conductor dividing $\mathfrak{c}$ and define

$$
\Sigma_{K}=\Sigma_{K}^{(1)} \cup \Sigma_{K}^{(2)} \subset \Sigma
$$

to be the disjoint union of the sets

$$
\begin{aligned}
& \Sigma_{K}^{(1)}=\left\{\psi \in \Sigma \text { of infinity type }\left(\kappa_{1}, \kappa_{2}\right), \kappa_{1} \leq 0, \kappa_{2} \geq 1\right\}, \\
& \Sigma_{K}^{(2)}=\left\{\psi \in \Sigma \text { of infinity type }\left(\kappa_{1}, \kappa_{2}\right), \kappa_{1} \geq 1, \kappa_{2} \leq 0\right\} .
\end{aligned}
$$


For all $\psi \in \Sigma_{K}, s=0$ is a critical point for the Hecke $L$-function $L\left(\psi^{-1}, s\right)$, and Katz's $p$-adic $L$-function is constructed by interpolating the (suitably normalized) values $L\left(\psi^{-1}, 0\right)$ as $\psi$ ranges over $\Sigma_{K}^{(2)}$.

More precisely, let $\hat{\Sigma}_{K}$ denote the completion of $\Sigma_{K}^{(2)}$ with respect to the compact open topology on the space of functions on a certain subset of $\mathbf{A}_{K}^{\times}$, as described in [3, Sect. 5.2]. By the work of Katz [23], there exists a $p$-adic analytic function

$$
L_{p}(K): \hat{\Sigma}_{K} \longrightarrow \mathbb{C}_{p}
$$

which is uniquely characterized by the following interpolation property: for all $\psi \in$ $\Sigma_{K}^{(2)}$ of infinity type $\left(\kappa_{1}, \kappa_{2}\right)$,

$$
L_{p}(K)(\psi)=\mathfrak{e}_{K}(\psi) \mathfrak{f}_{K}(\psi) \frac{\Omega_{p}^{\kappa_{1}-\kappa_{2}}}{\Omega^{\kappa_{1}-\kappa_{2}}} L_{\mathfrak{c}}\left(\psi^{-1}, 0\right),
$$

where

- $L_{\mathfrak{c}}\left(\psi^{-1}, s\right)$ is Hecke's $L$-function associated to $\psi^{-1}$ with the Euler factors at primes dividing $\mathfrak{c}$ removed,

- $\Omega_{p} \in \mathbb{C}_{p}^{\times}$is a $p$-adic period attached to $K$, as defined in [3, (140)], [4, (25)],

- $\Omega \in \mathbb{C}^{\times}$is the complex period associated to $K$ as defined in [3, (137)],

- $\mathfrak{e}_{K}(\psi)=\left(1-\frac{\psi(\wp)}{p}\right)\left(1-\psi^{-1}(\bar{\wp})\right)$, and $\mathfrak{f}_{K}(\psi)=\frac{\left(\kappa_{1}-1\right) ! \cdot D_{K}^{K_{2} / 2}}{(2 \pi)^{\kappa_{2}}}$.

The following result is commonly known as Katz's Kronecker $p$-adic limit formula. It computes the value of $L_{p}(K)$ at a finite order character $\psi$ of $G_{K}$, which lies outside the region of interpolation (cf. [14, Ch. II, Sect. 5.2], [17, p. 90], [23, Sects. 10.4, 10.5]):

$$
L_{p}(K)(\psi)=\mathfrak{f}_{p}(\psi) \cdot \log _{p}\left(u_{\psi^{-1}}\right)
$$

where

$$
\mathfrak{f}_{p}(\psi)=\left\{\begin{array}{ll}
\frac{1}{2}\left(\frac{1}{p}-1\right) & \text { if } \psi=1 \\
\frac{-1}{24 c}(1-\psi(\bar{\wp}))\left(1-\frac{\psi(\bar{\wp})}{p}\right) & \text { if } \psi \neq 1 .
\end{array},\right.
$$

Here $c>0$ is the smallest positive integer in the conductor ideal of $\psi$.

\section{Classical and $p$-adic Rankin $L$-functions}

\subsection{Eisenstein series}

Let $\chi:\left(\mathbb{Z} / N_{\chi} \mathbb{Z}\right)^{\times} \rightarrow \mathbb{C}$ be a Dirichlet character of conductor $N_{\chi}$ and let $\mathbb{Q}_{\chi}$ denote the finite extension of $\mathbb{Q}$ generated by the values of $\chi$. For any multiple $N$ of $N_{\chi}$, let $\chi_{N}$ denote the character $\bmod N$ induced by $\chi$.

For every positive integer $k \geq 1$, let $M_{k}\left(N, \chi_{N}\right)$ and $S_{k}\left(N, \chi_{N}\right)$ denote the spaces of holomorphic (resp. cuspidal) modular forms of weight $k$, level $N$, and character $\chi_{N}$.

We also let $M_{k}^{\mathrm{an}}\left(N, \chi_{N}\right)$ and $S_{k}^{\mathrm{an}}\left(N, \chi_{N}\right)$ denote the space of real analytic functions on the upper half plane with the same transformation properties under $\Gamma_{0}(N)$ and 
having bounded growth (resp. rapid decay) at the cusps. On these spaces, one may define the Shimura-Maass derivative operator

$$
\delta_{k}:=\frac{1}{2 \pi i}\left(\frac{d}{d z}+\frac{i k}{2 y}\right): M_{k}^{\mathrm{an}}\left(N, \chi_{N}\right) \longrightarrow M_{k+2}^{\mathrm{an}}\left(N, \chi_{N}\right) .
$$

For every $k \geq 1$ such that $\chi(-1)=(-1)^{k}$, define the nonholomorphic Eisenstein series of weight $k$ and level $N$ attached to the character $\chi_{N}$ as the function on $\mathcal{H} \times \mathbb{C}$ given by the rule

$$
\tilde{E}_{k, \chi_{N}}(z, s)=\sum_{(m, n) \in \mathbb{Z}^{2} \backslash\{(0,0)\}} \frac{\chi_{N}^{-1}(n)}{(m N z+n)^{k}} \cdot \frac{y^{s}}{|m N z+n|^{2 s}} .
$$

Although a priori this series only converges for $\Re(s)>1-k / 2$, it can be extended to a meromorphic function in the variable $s$ on the whole complex plane $\mathbb{C}$. For $k>2$, or $k \geq 1$ but $\chi \neq 1$, the series arising by setting $s=0$ is actually holomorphic in $z$ and gives rise to a modular form

$$
\tilde{E}_{k, \chi_{N}}(z):=\tilde{E}_{k, \chi_{N}}(z, 0) \in M_{k}(N, \chi)
$$

For any value of $s$, the series $\tilde{E}_{k, \chi_{N}}(z, s)$ belongs to $M_{k}^{\text {an }}(N, \chi)$ and one verifies that

$$
\delta_{k} \tilde{E}_{k, \chi_{N}}(z, s)=-\frac{s+k}{4 \pi} \tilde{E}_{k+2, \chi_{N}}(z, s-1) .
$$

Moreover, if we let $\delta_{k}^{t}=\delta_{k+2 t-2} \cdots \delta_{k+2} \delta_{k}$ denote the $t$-fold iterate of the ShimuraMaass operator, then for all $t \leq(k-1) / 2$ we have

$$
\tilde{E}_{k, \chi_{N}}(z,-t)=\frac{(k-2 t-1) !}{(k-t-1) !}(-4 \pi)^{t} \delta_{k-2 t}^{t} \tilde{E}_{k-2 t, \chi_{N}}(z) .
$$

Define a normalization $E_{k, \chi_{N}} \in M_{k}(N, \chi)$ of the Eisenstein series as

$$
E_{k, \chi_{N}}(z)=\frac{N^{k}(k-1) !}{2(-2 \pi i)^{k} \tau\left(\chi^{-1}\right)} \cdot \tilde{E}_{k, \chi_{N}}(z)
$$

where

$$
\tau(\chi)=\sum_{a=1}^{N_{\chi}} \chi(a) e^{\frac{2 \pi a i}{N_{\chi}}}
$$

is the Gauss sum associated to the Dirichlet character $\chi$. 
Let $\sigma_{k-1, \chi}$ denote the function on the positive integers defined as $\sigma_{k-1, \chi}(n):=$ $\sum_{d \mid n} \chi(d) d^{k-1}$. Then $E_{k, \chi}$ is a newform of level $N_{\chi}$ and its $q$-expansion is

$$
E_{k, \chi}(q)=\frac{L(\chi, 1-k)}{2}+\sum_{n=1}^{\infty} \sigma_{k-1, \chi}(n) q^{n} \in M_{k}\left(N_{\chi}, \chi\right), \quad q=e^{2 \pi i z}
$$

When $N>N_{\chi}, E_{k, \chi_{N}}(q)$ is a $\mathbb{Q}_{\chi}$-linear combination of the modular forms $E_{k, \chi}\left(q^{d}\right)$ as $d$ ranges over the positive divisors of $N / N_{\chi}$ (cf. [35, (3.3), (3.4)] for the precise expression). In particular, $E_{k, \chi_{N}}$ is an eigenform with respect to all good Hecke operators $T_{\ell}, \ell \nmid N$ with the same eigenvalues of $E_{k, \chi}$.

\subsection{Classical Rankin's $\boldsymbol{L}$-function}

Recall that the Petersson scalar product on the space of real analytic modular forms $S_{l}^{\mathrm{an}}(N, \chi) \times M_{l}^{\mathrm{an}}(N, \chi)$ is given by

$$
\left\langle f_{1}, f_{2}\right\rangle_{l, N}:=\int_{\Gamma_{0}(N) \backslash \mathcal{H}} y^{l} \overline{f_{1}(z)} f_{2}(z) \frac{d x d y}{y^{2}} .
$$

Let

$$
g_{l}=\sum_{n \geq 1} a_{n}\left(g_{l}\right) q^{n} \in S_{l}\left(N, \chi_{g}\right), \quad f_{k}=\sum_{n \geq 1} a_{n}\left(f_{k}\right) q^{n} \in M_{k}\left(N, \chi_{f}\right)
$$

be two eigenforms of weights $l>k \geq 1$ and nebentype characters $\chi_{g}$ and $\chi_{f}$, respectively. We do not assume $g_{l}$ and $f_{k}$ to be newforms, but we do assume them to be eigenvectors for all good and bad Hecke operators.

Set $\chi:=\left(\chi_{g} \chi_{f}\right)^{-1}$ and let $g_{l}^{*}=\sum_{n \geq 1} \bar{a}_{n}\left(g_{l}\right) q^{n} \in S_{l}\left(N, \chi_{g}^{-1}\right)$ denote the modular form whose fourier coefficients are the complex conjugates of those of $g_{l}$.

For a rational prime $q$, we let $\left(\alpha_{q}\left(g_{l}\right), \beta_{q}\left(g_{l}\right)\right)$ denote the pair of roots of the Hecke polynomial $X^{2}-a_{q}\left(g_{l}\right) X+\chi_{g, N}(q) q^{l-1}$ which we label in such a way that $\operatorname{ord}_{q}\left(\alpha_{q}\left(g_{l}\right)\right) \leq \operatorname{ord}_{q}\left(\beta_{q}\left(g_{l}\right)\right)$. Note that $\left(\alpha_{q}\left(g_{l}\right), \beta_{q}\left(g_{l}\right)\right)=\left(a_{q}\left(g_{l}\right), 0\right)$ when $q \mid N$. If the weight is $l=1$ and $q \nmid N$, then both $\alpha_{q}\left(g_{l}\right)$ and $\beta_{q}\left(g_{l}\right)$ are $q$-units; in that case, we just choose an arbitrary ordering of this pair. Adopt similar notations for $f_{k}$.

Define the Rankin L-function of the convolution of $g_{l}$ and $f_{k}$ as the Euler product

$$
L\left(g_{l} \otimes f_{k}, s\right)=\prod_{q} L^{(q)}\left(g_{l} \otimes f_{k}, s\right)
$$

where $q$ ranges over all prime numbers and

$$
\begin{aligned}
L^{(q)}\left(g_{l} \otimes f_{k}, s\right)= & \left(1-\alpha_{q}\left(g_{l}\right) \alpha_{q}\left(f_{k}\right) q^{-s}\right)^{-1}\left(1-\alpha_{q}\left(g_{l}\right) \beta_{q}\left(f_{k}\right) q^{-s}\right)^{-1} \\
& \times\left(1-\beta_{q}\left(g_{l}\right) \alpha_{q}\left(f_{k}\right) q^{-s}\right)^{-1}\left(1-\beta_{q}\left(g_{l}\right) \beta_{q}\left(f_{k}\right) q^{-s}\right)^{-1} .
\end{aligned}
$$


Proposition 3.1 (Shimura) For all $s \in \mathbb{C}$ with $\Re(s)>>0$, we have

$$
L\left(g_{l} \otimes f_{k}, s\right)=\frac{1}{2} \frac{(4 \pi)^{s}}{\Gamma(s)}\left\langle g_{l}^{*}(z), \tilde{E}_{l-k, \chi_{N}}(z, s-l+1) \cdot f_{k}(z)\right\rangle_{l, N}
$$

Choose integers $m, t$ such that

$$
l=k+m+2 t \quad \text { andset } \quad j=(l+k+m-2) / 2=l-t-1 .
$$

For $m \geq 1$ and $t \geq 0$, evaluating Eq. (19) at $s=j$ and using Eqs. (14) and (15), one finds that

$$
\mathfrak{f}_{\mathrm{HR}}(l, k, m) \cdot L\left(g_{l} \otimes f_{k}, j\right)=\left\langle g_{l}^{*}(z), \delta_{m}^{t} E_{m, \chi_{N}}(z) \cdot f_{k}(z)\right\rangle_{l, N},
$$

where

$$
\mathfrak{f}_{\mathrm{HR}}(l, k, m)=\frac{(-1)^{t}(m+t-1) !(j-1) !(i N)^{m}}{2^{l-1}(2 \pi)^{l+m-1} \cdot \tau\left(\chi^{-1}\right)} .
$$

\subsection{Critical values, algebraicity, and the Hida-Rankin $p$-adic $L$-function}

Since we are assuming $l>k \geq 1$, an integer $j$ is critical for $L\left(g_{l} \otimes f_{k}, s\right)$ if and only if $j \in[k, l-1]$. We shall restrict our attention to critical integers in the range

$$
j \in\left[\frac{l+k-1}{2}, l-1\right]
$$

and for a given such $j$ we set

$$
t:=l-j-1 \text { and } m:=l-k-2 t .
$$

From Eq. (20), it follows that

$$
\mathfrak{f}_{\mathrm{HR}}(l, k, m) \cdot L\left(g_{l} \otimes f_{k}, j\right)=\left\langle g_{l}^{*}(z), \delta_{m}^{t} E_{m, \chi_{N}}(z) \cdot f_{k}(z)\right\rangle_{l, N} .
$$

Define the algebraic part of $L\left(g_{l} \otimes f_{k}, j\right)$ as in [5, (9)]:

$$
L^{\mathrm{alg}}\left(g_{l} \otimes f_{k}, j\right):=\mathfrak{f}_{\mathrm{HR}}(l, k, j) \frac{L\left(g_{l} \otimes f_{k}, j\right)}{\left\langle g_{l}^{*}, g_{l}^{*}\right\rangle_{l, N}}=\frac{\left\langle g_{l}^{*}(z), \delta_{m}^{t} E_{m, \chi_{N}}(z) \cdot f_{k}(z)\right\rangle_{l, N}}{\left\langle g_{l}^{*}, g_{l}^{*}\right\rangle_{l, N}}
$$

Fix a prime $p \nmid N$ at which $g_{l}$ is ordinary and let $g_{l, \alpha} \in S_{l}\left(N p, \chi_{g}\right)$ denote the ordinary $p$-stabilization of $g_{l}$ on which $U_{p}$ acts with eigenvalue $\alpha_{p}\left(g_{l}\right)$.

Let $\mathbf{g}$ be a Hida family of ordinary overconvergent modular forms of tame (but not necessarily primitive) level $N$, passing through $g_{l, \alpha}$. The Hida family is parametrized by a finite étale rigid analytic cover $U_{\mathbf{g}}$ of weight space $\mathcal{W}$. By shrinking $U_{\mathbf{g}}$ if necessary, we assume that $U_{\mathbf{g}}\left(\mathbb{Z}_{p}\right)$ is fibered over a single residue class modulo $p-1$ of $\mathcal{W}\left(\mathbb{Z}_{p}\right)=\mathbb{Z}_{p}^{\times} \simeq \mathbb{Z} /(p-1) \mathbb{Z} \times \mathbb{Z}_{p}$. By a slight abuse of notation which shall be 
harmless for our purposes, we identify throughout points in $U_{\mathbf{g}}$ with their image in $\mathcal{W}$ under the weight map.

With these conventions, for every classical weight $l \in U_{\mathbf{g}} \cap \mathbb{Z}^{\geq 2}$ we let

$$
g_{l} \in S_{l}\left(N, \chi_{g}\right)
$$

denote the classical cusp form whose ordinary $p$-stabilization is the specialization of $\mathbf{g}$ at an arithmetic point in $U_{\mathbf{g}}$ of weight $l$.

Define

$$
\mathfrak{e}_{\mathrm{HR}}(l, k, j):=\frac{\mathcal{E}\left(g_{l}, f_{k}, j\right)}{\mathcal{E}_{1}\left(g_{l}\right) \mathcal{E}_{0}\left(g_{l}\right)},
$$

where

$$
\begin{aligned}
\mathcal{E}\left(g_{l}, f_{k}, j\right)= & \left(1-\beta_{p}\left(g_{l}\right) \alpha_{p}\left(f_{k}\right) p^{t-l+1}\right)\left(1-\beta_{p}\left(g_{l}\right) \beta_{p}\left(f_{k}\right) p^{t-l+1}\right) \\
& \times\left(1-\beta_{p}\left(g_{l}\right) \alpha_{p}\left(f_{k}\right) \chi(p) p^{t-l+1}\right)\left(1-\beta_{p}\left(g_{l}\right) \beta_{p}\left(f_{k}\right) \chi(p) p^{t-l+1}\right), \\
\mathcal{E}_{1}\left(g_{l}\right)= & 1-\beta_{p}\left(g_{l}\right)^{2} p^{-l}, \\
\mathcal{E}_{0}\left(g_{l}\right)= & 1-\beta_{p}\left(g_{l}\right)^{2} p^{1-l} .
\end{aligned}
$$

In [20, Sect. 7.4], Hida constructed a three-variable $p$-adic $L$-function interpolating central critical values of the Rankin $L$-function associated to the convolution of two Hida families of modular forms. For the purposes of this note, it will suffice to retain the restriction of this $p$-adic $L$-function to the one-dimensional domain afforded by $U_{\mathbf{g}}$. Here we will work with the notations and normalizations adopted in [5]. In order to introduce this $p$-adic $L$-function properly, we shall make use of the following operators on the space of overconvergent $p$-adic modular forms that we introduce here by describing their action on $q$-expansions:

- Serre's derivative operator $d=q \cdot \frac{d}{d q}$, which may be regarded as the $p$-adic avatar of the Shimura-Maass operator invoked above,

- The $U$ and $V$ operators acting on a modular form $\phi=\sum a_{n} q^{n}$ by the rules

$$
U(\phi)=\sum a_{p n} q^{n} \text { and } V(\phi)=\sum a_{n} q^{p n},
$$

- Hida's ordinary idempotent $e_{\text {ord }}:=\lim U_{p}^{n !}$, and

- $p$-depleting operator: $\phi^{[p]}:=(1-U V)(\phi)=\sum_{p \nmid n} a_{n} q^{n}$.

Let $\mathcal{E}$ denote the Kuga-Sato variety fibered over $X_{1}(N)$ and let $\mathcal{E}^{l-2}$ denote the fiber product of $l-2$ copies of $\mathcal{E}$ over $X_{1}(N)$. The dimension of $\mathcal{E}^{l-2}$ is $l-1$ and the middle de Rham cohomology group $H_{\mathrm{dR}}^{l-1}\left(\mathcal{E}^{l-2} / \mathbb{C}_{p}\right)$ contains the canonical regular differential form $\omega_{g_{l}^{*}}$ associated to $g_{l}^{*}$. Let

$$
\langle\rangle: H_{\mathrm{dR}}^{l-1}\left(\mathcal{E}^{l-2} / \mathbb{C}_{p}\right) \times H_{\mathrm{dR}}^{l-1}\left(\mathcal{E}^{l-2} / \mathbb{C}_{p}\right) \longrightarrow \mathbb{C}_{p}
$$

denote the nondegenerate Poincaré pairing on $H_{\mathrm{dR}}^{l-1}\left(\mathcal{E}^{l-2} / \mathbb{C}_{p}\right)$. 
The $g_{l}^{*}$-isotypic component of $H_{\mathrm{dR}}^{l-1}\left(\mathcal{E}^{l-2} / \mathbb{C}_{p}\right)$ is two-dimensional over $\mathbb{C}_{p}$ and it admits a one-dimensional unit root subspace, denoted $H_{\mathrm{dR}}^{l-1}\left(\mathcal{E}^{l-2} / \mathbb{C}_{p}\right)^{\mathrm{u}-\mathrm{r}}$, on which the Frobenius endomorphism acts as multiplication by a $p$-adic unit. This unit root subspace is complementary to the line spanned by $\omega_{g_{l}^{*}}$. There is thus a single class $\eta_{g_{l}^{*}}^{\mathrm{u}-\mathrm{r}} \in H_{\mathrm{dR}}^{l-1}\left(\mathcal{E}^{l-2} / \mathbb{C}_{p}\right)^{\mathrm{u}-\mathrm{r}}$ satisfying

$$
\left\langle\omega_{g_{l}^{*}}, \eta_{g_{l}^{*}}^{\mathrm{u}-\mathrm{r}}\right\rangle=1
$$

Let $\Lambda_{g}$ denote the algebra of Iwasawa functions on $U_{\mathbf{g}}$ and let $\mathcal{K}_{\mathbf{g}}$ denote the fraction field of $\Lambda_{\mathbf{g}}$. As shown in [5], there exists a unique $p$-adic $L$-function $L_{p}(\mathbf{g}, f) \in \mathcal{K}_{\mathbf{g}}$ satisfying

$$
L_{p}(\mathbf{g}, f)(l)=\frac{1}{\mathcal{E}_{0}\left(g_{l}, f_{k}, j\right)}\left\langle\eta_{g_{l}^{*}}^{u r}, e_{\mathrm{ord}}\left(d^{t} E_{m, \chi_{N}}^{[p]} \cdot f_{k}\right)\right\rangle
$$

for all $l \in U_{\mathbf{g}} \cap \mathbb{Z}_{\geq 2}$. Note that $d^{t} E_{m, \chi_{N}}^{[p]} \cdot f_{k}$ is an overconvergent modular form of weight $l \geq 2$ and hence its ordinary projection is classical by a celebrated theorem of Hida and Coleman. This way $e_{\mathrm{ord}}\left(d^{t} E_{m, \chi_{N}}^{[p]} \cdot f_{k}\right)$ gives rise to a regular differential form in $H_{\mathrm{dR}}^{l-1}\left(\mathcal{E}^{l-2} / \mathbb{C}_{p}\right)$, which may therefore be written as

$$
\begin{aligned}
e_{\text {ord }}\left(d^{t} E_{m, \chi_{N}}^{[p]} \cdot f_{k}\right)= & C\left(g_{l}, f_{k}\right) \cdot \omega_{g_{l}^{*}} \\
& +(\text { Other terms with respect to an orthogonal basis }) .
\end{aligned}
$$

In plane terms, the above formula might be read as

$$
L_{p}(\mathbf{g}, f)(l)=\frac{C\left(g_{l}, f_{k}\right)}{\mathcal{E}_{0}\left(g_{l}, f_{k}, j\right)}
$$

The $p$-adic $L$-function $L_{p}\left(\mathbf{g}, f_{k}\right)$ deserves its name because it obeys and it is characterized by the following interpolation formula that relates the values of $L_{p}\left(\mathbf{g}, f_{k}\right)$ at integers $l>k$ to critical values of a Rankin $L$-function. In fact, it follows from (23) and (25) that for every $l \in U_{\mathbf{g}} \cap \mathbb{Z}_{l>k}$ we have

$$
L_{p}\left(\mathbf{g}, f_{k}\right)(l)=\mathfrak{e}_{\mathrm{HR}}(l, k, j) \cdot L^{\mathrm{alg}}\left(g_{l} \otimes f_{k}, j\right)=\mathfrak{e}_{\mathrm{HR}}(l, k, j) \mathfrak{f}_{\mathrm{HR}}(l, k, j) \frac{L\left(g_{l} \otimes f_{k}, j\right)}{\left\langle g_{l}^{*}, g_{l}^{*}\right\rangle_{l, N}} .
$$

We call this function the Hida-Rankin p-adic L-function associated to $\mathbf{g}$ and $f_{k}$.

Note that $l=1$ lies outside the above region of interpolation. Assume that there exists an eigenform $g_{1} \in M_{1}\left(N_{g}, \chi_{g}\right)$ such that the ordinary $p$-stabilization $g_{1, \alpha}$ arises as the specialization of $\mathbf{g}$ at an arithmetic point of weight 1 in $U_{\mathbf{g}}$. Notice that this is not always the case, as a Hida family may in general specialize to nonclassical overconvergent modular forms at points of weight one.

The following result provides a formula for the value of $L_{p}(\mathbf{g}, f)$ at $l=1$ and was proved in [10, Sect. 2]. Assume that $k=2$ and $\chi_{f}=1$, so that $\chi=\chi_{g}^{-1}$, and set $f=f_{2}$. Recall the $p$-adic iterated integrals introduced in (5). 
Proposition 3.2 Let $h:=E_{1, \chi_{N}} \in M_{1}\left(N, \chi_{N}\right)$. Then $L_{p}(\mathbf{g}, f)$ has no pole at $l=1$ and

$$
L_{p}(\mathbf{g}, f)(1)=\int_{\gamma_{g_{1}}} f \cdot h .
$$

Proof Combine [10, Proposition 2.6] and [12, Remark 4.5, Proposition 4.6].

\subsection{Bertolini-Darmon-Prasanna's $p$-adic $L$-function}

As in the introduction, let $E / \mathbb{Q}$ be an elliptic curve of conductor $N_{E}$ and let $f \in$ $S_{2}\left(N_{E}\right)$ denote the eigenform associated to it by modularity. As in Sect. 2.1, let also $K$ be an imaginary quadratic field of discriminant $-D_{K} \leq-7$ fulfilling the Heegner hypothesis.

Let $\mathfrak{c} \subseteq \mathcal{O}_{K}$ be an integral ideal and set $N=\operatorname{lcm}\left(N_{E}, D_{K} N_{K / \mathbb{Q}}(\mathfrak{c})\right)$. Let $\Sigma$ denote the set of Hecke characters of $K$ of conductor dividing $\mathfrak{c}$. For any Hecke character $\psi \in \Sigma$ of infinity type $\left(\kappa_{1}, \kappa_{2}\right)$, let $L(f, \psi, s)$ denote the $L$-function associated to the compatible system of Galois representations afforded by the tensor product $\varrho_{f \mid G_{K}} \otimes \psi$ of the (restriction to $G_{K}$ of) the Galois representations attached to $f$ and the character $\psi$.

As usual, $L(f, \psi, s)=\prod_{q} L^{(q)}\left(q^{-s}\right)$ is defined as a product of Euler factors ranging over the set of prime numbers. The Euler factors at the primes $q$ such that $q \nmid N$ are exactly the same as those of the Rankin L-series $L\left(\theta_{\psi} \otimes f, s\right)$ introduced above, but may differ at the primes $q$ such that $q \mid N$ (details can be found in [16] for $f$ modular form of weight 2).

Let $\Sigma_{f, K} \subset \Sigma$ be the subset of Hecke characters of trivial central character in $\Sigma$ for which $L\left(f, \psi^{-1}, s\right)$ is self-dual and $s=0$ is its central critical point. This set is naturally the disjoint union of the three subsets:

$$
\begin{gathered}
\Sigma_{f, K}^{(1)}=\left\{\psi \in \Sigma_{f, K} \text { of infinity type }(1,1)\right\}, \\
\Sigma_{f, K}^{(2)}=\left\{\psi \in \Sigma_{f, K} \text { of infinity type }(2+\kappa,-\kappa), \kappa \geq 0\right\}
\end{gathered}
$$

and

$$
\Sigma_{f, K}^{\left(2^{\prime}\right)}=\left\{\psi \in \Sigma_{f, K} \text { of infinity type }(-\kappa, \kappa+2), \kappa \geq 0\right\}
$$

Fix a prime $p=\wp \bar{\wp}$ that splits in $K$. Each of the three sets $\Sigma_{f, K}^{(1)}, \Sigma_{f, K}^{(2)}$, and $\Sigma_{f, K}^{\left(2^{\prime}\right)}$ are dense in the completion $\hat{\Sigma}_{f, K}$ of $\Sigma_{f, K}$ with respect to the $p$-adic compact open topology as explained in [3, Sect. 5.2]. As shown in [3], there exists a unique $p$-adic analytic function

$$
L_{p}(f, K): \hat{\Sigma}_{f, K} \longrightarrow \mathbb{C}_{p}
$$

interpolating the critical values $L\left(f, \psi^{-1}, 0\right)$ for $\psi \in \Sigma_{f, K}^{(2)}$, suitably normalized. 
We refer to $L_{p}(f, K)$ as the Bertolini-Darmon-Prasanna $p$-adic Rankin $L$-function attached to the pair $(f, K)$.

Consider a character $\Psi \in \Sigma_{f, K}^{(2)}$ of type $(2+\kappa,-\kappa)$, for $\kappa \geq 0$. According to [3, Sect. 5.2], the interpolation formula for $L_{p}(f, K)$ reads

$$
L_{p}(f, K)(\Psi)=\mathcal{E}_{c} \cdot \mathfrak{e}_{\mathrm{BDP}}(\Psi) \cdot \mathfrak{f}_{\mathrm{BDP}}(\Psi) \cdot \frac{\Omega_{p}^{4 \kappa+4}}{\Omega^{4 \kappa+4}} \cdot L\left(f, \Psi^{-1}, 0\right),
$$

where

- $\mathcal{E}_{c}=\prod_{q \mid c} \frac{q-\chi_{K}(q)}{q-1}, \quad \mathfrak{e}_{\mathrm{BDP}}(\Psi)=\left(1-a_{p}(f) \Psi^{-1}(\bar{\wp})+p \Psi^{-1}(\bar{\wp})^{2}\right)^{2}$,

- $\mathfrak{f}_{\mathrm{BDP}}(\Psi)=\left(\frac{2 \pi}{\sqrt{D_{K}}}\right)^{2 \kappa+1} \kappa !(\kappa+1) ! \cdot 2^{\sharp q \mid\left(D_{K}, N_{E}\right)} \cdot \omega(f, \Psi)^{-1}$

with $\omega(f, \Psi)$ as defined in [3, (5.1.11)].

If $\psi$ is a finite order anticyclotomic character of conductor $c \mid \mathfrak{c}$, then $\psi \mathbf{N}_{K}$ lies outside the region of interpolation and the main theorem of [4] asserts that

$$
L_{p}(f, K)\left(\psi^{-1} \mathbf{N}_{K}\right)=\mathfrak{f}_{p}(f, \psi) \times \log _{\omega_{E}}\left(P_{\psi}\right)^{2},
$$

where $\mathfrak{f}_{p}(f, \psi)=\left(1-\psi(\bar{\wp}) p^{-1} a_{p}(f)+\psi^{2}(\bar{\wp}) p^{-1}\right)^{2}$.

\section{Proof of the main theorem}

Recall the three eigenforms that have been fixed at the outset:

$$
f \in S_{2}\left(N_{E}\right), \quad g=\theta_{\psi} \in M_{1}\left(D_{K} c^{2}, \chi_{K}\right)_{\mathbb{Q}_{\psi}}, \quad h=\mathrm{E}_{1, \chi_{K}} \in M_{1}\left(D_{K}, \chi_{K}\right) .
$$

Choose and fix modular forms $\breve{f} \in S_{2}(N)[f]$ and $\breve{g} \in M_{1}\left(N, \chi_{K}\right)[g]$ that are eigenforms for all good and bad Hecke operators. For the sake of concreteness, we may write

$$
\breve{f}(z)=\sum_{d \mid \frac{N}{N_{E}}} \mu_{d}(f) f(d z), \quad \breve{g}(z)=\sum_{d \mid \frac{N}{D c^{2}}} \mu_{d}(g) g(d z),
$$

where $\mu_{d}(f), \mu_{d}(g)$ belong to the number field $\mathbb{Q}_{\psi}\left(f_{N}\right)$ introduced in the paragraph preceding Theorem 1.4.

Fix a prime $p \nmid N$ that splits in $K$ and choose a root $\alpha$ of $T^{2}-a_{p}(g) T+1$. As in the introduction, let $\breve{g}_{\alpha} \in M_{1}\left(N p, \chi_{K}\right)$ denote the $p$-stabilization of $\breve{g}$ on which $U_{p}$ acts with eigenvalue $\alpha$.

There exists a unique $p$-adic Hida family $\mathbf{g}$ of theta series of tame level $D_{K} c^{2}$ and tame character $\chi_{K}$ passing through $g_{\alpha}$. As in Sect. 3.3 and (24), for every classical weight $l \in U_{\mathbf{g}} \cap \mathbb{Z}^{\geq 2}$ we let

$$
g_{l} \in S_{l}\left(D_{K} c^{2}, \chi_{K}\right)
$$

denote the classical newform whose ordinary $p$-stabilization is the specialization of $\mathbf{g}$ at an arithmetic point in $U_{\mathbf{g}}$ of weight $l$. Notice that at $l=1$ the modular form $g_{l}$ 
is still classical, by assumption, but it might not be a cusp form. In this case we have $g_{1}=g \in M_{1}\left(D_{k} c^{2}, \chi_{K}\right)$.

For every such $l$, we can also explicitly describe the Hecke character $\psi_{l-1}$ of conductor $c$ and infinity type $(0, l-1)$ such that $g_{l}=\theta_{\psi_{\ell-1}}$. We do the same construction done in [20, p. 235-236] and [10, Sect. 3], but we slightly change conventions. Pick a $p$-adic unitary character $\lambda$ of conductor $c \bar{\varnothing}$ and infinity type $(0,1)$. Then define $\psi_{\ell-1}(\mathfrak{q}):=\psi(\mathfrak{q})\langle\lambda(\mathfrak{q})\rangle^{\ell-1}$, and define $\psi_{\ell-1}(\bar{\wp}):=p^{\ell-1} / \psi_{\ell-1}(\wp)$. At any prime $q=\mathfrak{q} \overline{\mathfrak{q}}$ which splits in $K$, we have

$$
\alpha_{q}\left(g_{l}\right)=\psi_{l-1}(\mathfrak{q}), \quad \beta_{q}\left(g_{l}\right)=\psi_{l-1}(\overline{\mathfrak{q}}) .
$$

Our running hypothesis on $p$ and the Heegner assumption imply that this is the case for $q=p$ and for any of the primes dividing $N$ but not $D_{K}$. forms

Together with $\mathbf{g}$, it will also be useful to consider the $\Lambda$-adic family of modular

$$
\breve{\mathbf{g}}(q)=\sum_{d \mid \frac{N}{D c^{2}}} \mu_{d}(g) \mathbf{g}\left(q^{d}\right)
$$

arising from our choice of $\breve{g}$. Note that $\breve{\mathbf{g}}$ specializes to $\breve{g}_{\alpha}$ in weight one.

Let $U_{\mathbf{g}}^{\circ}$ denote the subset of $U_{\mathbf{g}}$ consisting of classical points of weights of the form $2 l+3 \equiv 1(\bmod p-1)$ with $l \in \mathbb{Z}_{\geq 1}$. According to the conventions about Hida families adopted in Sect. 3.3, note that $U_{\mathbf{g}}^{\circ}$ is dense in $U_{\mathbf{g}}$.

Set $j=l+2, t=l$, and $m=1$. Then the interpolation formula of (26) at points in $U_{\mathbf{g}}^{\circ}$ reads as follows:

$$
L_{p}(\breve{\mathbf{g}}, \breve{f})(2 l+3, l+2)=\mathfrak{e}_{\mathrm{HR}}(l) \cdot \mathfrak{f}_{\mathrm{HR}}(l) \cdot \frac{L\left(\breve{g}_{2 l+3} \otimes \breve{f}, l+2\right)}{\left\langle\breve{g}_{2 l+2}^{*}, \breve{g}_{2 l+2}^{*}\right\rangle_{l, N}},
$$

where $\mathfrak{e}_{\mathrm{HR}}(l)=\mathcal{E}(2 l+3,2, l+2) /\left(\mathcal{E}_{1}(2 l+3) \mathcal{E}_{0}(2 l+3)\right)$ with

$$
\begin{aligned}
\mathcal{E}(2 l+3,2, l+2) & =\left(1-\alpha_{f} \beta_{\theta_{2 l+3}} p^{-(l+2)}\right)^{2}\left(1-\beta_{f} \beta_{\theta_{2 l+3}} p^{-(l+2)}\right)^{2}, \\
\mathcal{E}_{1}(2 l+3) & =1-\beta_{\theta_{2 l+3}}^{2} p^{-2 l-3} \\
\mathcal{E}_{0}(2 l+3) & =1-\beta_{\theta_{2 l+3}}^{2} p^{-2 l-2}
\end{aligned}
$$

and

$$
\mathfrak{f}_{\mathrm{HR}}(l)=\frac{(-1)^{l} l !(l+1) ! \cdot i \cdot N}{2^{4 l+5} \pi^{2 l+3} \cdot \tau\left(\chi_{K}\right)}=(-1)^{l} \cdot \frac{l !(l+1) ! \cdot N}{2^{4 l+5} \pi^{2 l+3} \cdot \sqrt{D_{K}}} .
$$

Here the last equality holds because $\tau\left(\chi_{K}\right)=i \sqrt{D_{K}}$ (cfr. [32]).

We now need the following two basic lemmas.

Lemma 4.1 There exists a meromorphic function $\mathcal{E} u l_{N}(s)$ such that the following factorization formula holds

$$
L(\breve{g} \otimes \breve{f}, s)=\mathcal{E} u l_{N}(s) \cdot L(f, \psi, s)
$$

and $\mathcal{E} u l_{N}(1) \in \mathbb{Q}_{\psi}\left(f_{N}\right)^{\times}$. 
Proof Since we choose $\breve{f}$ and $\breve{g}$ to be eigenforms for all Hecke operators, we can use Euler products to compare the two L-functions. Their Euler factors are equal outside primes $q \mid N$. Then the factor $\mathcal{E} u l_{N}(s)$ is the product of the bad Euler factors at $q \mid N$ which encode this discrepancy. If we use the definitions of Eq. (18) and of [16, equation (20.2)], we find

$$
\begin{aligned}
& \mathcal{E}_{u l_{N}(s)} \\
& =\frac{\prod_{q \mid\left(N_{E}, D_{K}\right)}\left(1+q^{-s}\right) \prod_{q \| \mid N_{E}, q \nmid D_{K}}\left(1-a_{q}(f) q^{-s}\right)^{2} \prod_{q \mid D_{K}, q \nmid N_{E}}\left(1-a_{q}(f) a_{q}(g) q^{-s}+q^{1-2 s}\right)}{\prod_{q \mid N}\left(1-\alpha_{q}(\breve{f}) \alpha_{q}(\breve{g}) q^{-s}\right)}
\end{aligned}
$$

so that $\mathcal{E} u l_{N}(1)$ is a finite product of nonzero terms which lies in $\mathbb{Q}_{\psi}\left(f_{N}\right)$.

Lemma 4.2 For $l=-1$ and $l \in U_{\mathbf{g}}^{\circ}$, let $\Psi_{l}$ be the Hecke character $\Psi_{l}=$ $\left(\psi_{2 l+2}\right)^{-1} \mathbf{N}^{l+2}$ of conductor $c$ and infinity type $(l+2,-l)$. Then there exists a number $\mathcal{E}_{u l}^{H R}(l) \in \mathbb{Q}_{\psi}\left(f_{N}\right)$ such that the following equality of critical L-values holds:

$$
L\left(\breve{g}_{2 l+3} \otimes \breve{f}, l+2\right)=\mathcal{E} u l_{N}^{H R}(l) \cdot L\left(f, \Psi_{l}^{-1}, 0\right) .
$$

Moreover, for $l=-1$ we have $\mathcal{E} u l_{N}^{\mathrm{HR}}(-1) \neq 0$.

Proof The $L$-functions $L\left(\breve{g}_{2 l+3} \otimes \breve{f}, s\right)$ and $L\left(g_{2 l+3} \otimes f, l+2\right)$ are defined by an Euler product with exactly the same local factors at all primes $q$ except possible for the primes $q \mid N$. From the definition of L-factors, we have that $\mathcal{E} u l_{N}^{\mathrm{HR}}(l)$ lies in $\mathbb{Q}_{\psi}\left(f_{N}\right)$ for all $l$.

For $l=-1$, we have $\mathcal{E} u l_{N}^{H R}(-1)=\mathcal{E} u l_{N}(1) \in \mathbb{Q}_{\psi}\left(f_{N}\right)^{\times}$by Lemma 4.1.

Secondly, we have the following classical formula for the Petersson product, due essentially to Petersson (cf. [19, Theorem 5.1], [29, Satz 6]):

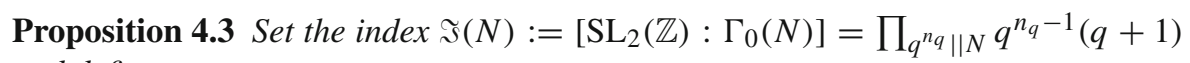
and define

$$
\mathfrak{f}_{\text {Pet }}(l):=\frac{\Im(N)}{\Im\left(D_{K} c^{2}\right)} \cdot \frac{(2 l+2) !}{2^{4 l+4} \pi^{2 l+3}} \cdot \frac{h_{c} \cdot \sqrt{D_{K} c^{2}}}{w_{c}},
$$

where $h_{c}$ and $w_{c}$ are the class number and the number of roots of unity of the order $\mathcal{O}_{c}$ of conductor $c$, respectively. Then

$$
\left\langle g_{2 l+3}^{*}, g_{2 l+3}^{*}\right\rangle_{N}=\mathfrak{f}_{P e t}(l) \cdot L\left(\psi_{2 l+2}^{2}, 2 l+3\right) .
$$

Let us introduce now the Hecke character $\Phi_{l}=\psi_{2 l+2}^{-2} \mathbf{N}^{2 l+3}$ of infinity type $(2 l+$ $3,-2 l-1)$, and note that $\Phi_{l}$ lies in the region of interpolation for the Katz $p$-adic $L$-function. Since $L\left(\Phi_{l}^{-1}, s\right)=L\left(\psi_{2 l+2}^{2}, s+2 l+3\right)$, it follows from (35) that

$$
\begin{aligned}
\left\langle\breve{g}_{2 l+3}^{*}, \breve{g}_{2 l+3}^{*}\right\rangle_{l, N} & =\mathcal{E} u l_{N}^{P e t}(l) \cdot\left\langle g_{2 l+3}, g_{2 l+3}\right\rangle_{l, N} \\
& =\mathcal{E} u l_{N}^{P e t}(l) \cdot \mathfrak{f}_{\operatorname{Pet}}(l) \cdot L\left(\Phi_{l}^{-1}, 0\right),
\end{aligned}
$$


where $\mathcal{E} u l_{N}^{P e t}(l) \in \mathbb{Q}_{\psi}$ is a nonzero number arising from the discrepancy at the primes $q \mid N$ of the local Hecke polynomials of $g_{2 l+3}$ and $\breve{g}_{2 l+3}$.

Set

$$
\mathcal{E}_{u} l_{N}(l)=\frac{\mathcal{E} u l_{N}^{\mathrm{HR}}(l)}{\mathcal{E}_{c} \cdot \mathcal{E} u l_{N}^{P e t}(l)} \quad \text { and } \quad \mathfrak{f}_{\infty}(l):=\frac{\mathfrak{f}_{\mathrm{HR}}(l) \cdot \mathfrak{f}_{K}\left(\Phi_{l}\right)}{\mathfrak{f}_{\mathrm{BDP}}\left(\Psi_{l}\right) \cdot \mathfrak{f}_{P e t}(l)}
$$

and define the function

$$
\mathfrak{f}: U_{\mathbf{g}}^{\circ} \longrightarrow \mathbb{C}_{p}, \quad \mathfrak{f}(l):=\mathcal{E} u l_{N}(l) \cdot \mathfrak{f}_{\infty}(l) .
$$

Theorem 4.4 The function $\mathfrak{f}$ interpolates to a $p$-adic analytic function on $U_{\mathbf{g}}$ and the following factorization of p-adic L-series holds:

$$
L_{p}(\breve{\mathbf{g}}, \breve{f})(2 l+3) \times L_{p}(K)\left(\Phi_{l}\right)=\mathfrak{f}(l) \cdot L_{p}(f, K)\left(\Psi_{l}\right)
$$

Proof In our setting, the Bertolini-Darmon-Prasanna interpolation formula reads as

$$
\begin{aligned}
L\left(f, \Psi_{l}^{-1}, 0\right) & =\frac{1}{\mathfrak{e}_{\mathrm{BDP}}\left(\Psi_{l}\right) \cdot \mathfrak{f}_{\mathrm{BDP}}\left(\Psi_{l}\right) \cdot \mathcal{E}_{c}} \cdot \frac{\Omega^{4 l+4}}{\Omega_{p}^{4 l+4}} \cdot L_{p}(f, K)\left(\Psi_{l}\right), \\
\mathfrak{e}_{\mathrm{BDP}}\left(\Psi_{l}\right) & =\left(1-a_{p}(f) \psi_{2 l+2}(\bar{\wp}) p^{-2-l}+\psi_{2 l+2}(\bar{\wp})^{2} p^{-2 l-3}\right)^{2}, \\
\mathfrak{f}_{\mathrm{BDP}}\left(\Psi_{l}\right) & =\left(\frac{2 \pi}{c \sqrt{D_{K}}}\right)^{2 l+1} l !(l+1) ! \cdot 2^{\sharp q \mid\left(D_{K}, N_{E}\right)} \cdot \omega\left(f, \Psi_{l}\right)^{-1}, \\
\mathcal{E}_{c} & =\prod_{q \mid c} \frac{q-\chi_{K}(q)}{q-1} .
\end{aligned}
$$

After replacing $k_{1}=2 l+3$ and $k_{2}=-2 l-1$ in the Katz interpolation formula, we find

$$
\begin{aligned}
L\left(\Phi_{l}^{-1}, 0\right) & =\frac{1}{\mathfrak{e}_{K}\left(\Phi_{l}\right) \cdot \mathfrak{f}_{K}\left(\Phi_{l}\right)} \cdot \frac{\Omega^{4 l+4}}{\Omega_{p}^{4 l+4}} \cdot L_{p}(K)\left(\Phi_{l}\right), \\
\mathfrak{e}_{K}\left(\Phi_{l}\right) & =\left(1-\psi_{2 l+2}^{-2}(\wp) p^{2 l+2}\right)\left(1-\frac{\psi_{2 l+2}^{2}(\bar{\wp})}{p^{2 l+3}}\right), \\
\mathfrak{f}_{K}\left(\Phi_{l}\right) & =\left(\frac{2 \pi}{\sqrt{D_{K}}}\right)^{2 l+1}(2 l+2) !
\end{aligned}
$$

Subsequently, substitute Eqs. (35), (37), and (38) into Eq. (32). Notice that by Eq. (31) we have $\mathfrak{e}_{\mathrm{HR}}(l) \mathfrak{e}_{K}(l)=\mathfrak{e}_{\mathrm{BDP}}(l)$. An elementary manipulation immediately shows that the decomposition formula (36) holds at all the points in $U_{\mathbf{g}}^{\circ}$.

Since $U_{\mathbf{g}}^{\circ}$ is dense in $U_{\mathbf{g}}$, in order to complete the proof of the theorem it only remains to prove the claim that $\mathfrak{f}$ extends to a $p$-adic analytic function on $U_{\mathbf{g}}$. 
Recall that $\mathfrak{f}(l)$ was defined as the product of two functions $\mathcal{E} u l_{N}(l)$ and $\mathfrak{f}_{\infty}(l)$. The Euler factors encoded in $\mathcal{E}_{u l_{N}}(l)$ are rational functions on powers of primes $q \mid N$ and hence $\mathcal{E} u l_{N}$ extends to a $p$-adic analytic function on $U_{\mathbf{g}}$.

As for the function $\mathfrak{f}_{\infty}(l)$, we first notice that from [3, Eq. (5.1.11)] we can compute that $\omega\left(f, \Psi_{l}\right)=(-1 / N)^{l+1} \cdot \psi_{2 l+2}(\mathfrak{N})^{-1}$. Hence $\omega\left(f, \Psi_{l}\right)$ also interpolates to $p$-adic analytic function on $U_{\mathbf{g}}$. Combining the explicit recipes given in the text for $\mathfrak{f}_{\mathrm{HR}}(l)$, $\mathfrak{f}_{K}\left(\Phi_{l}\right), \mathfrak{f}_{\mathrm{BDP}}\left(\Psi_{l}\right)$, and $\mathfrak{f}_{\text {Pet }}(l)$, one readily checks that

$$
\mathfrak{f}_{\infty}(l)=-\frac{\Im\left(D_{K} c^{2}\right)}{\Im(N)} \cdot \frac{N \cdot 2^{-\sharp q \mid\left(D_{K}, N_{E}\right)}}{h_{c} \cdot D_{K}} \cdot \frac{\psi_{2 l+2}(\mathfrak{N})}{c^{-2 l} \cdot N^{l+1}} .
$$

From this explicit description the claim follows.

Thanks to the above result, we can already prove Theorem 1.4. We evaluate (36) at the point of weight one in $U_{\mathbf{g}}$ arising when we set $l=-1$. In this case, Theorem 4.4 asserts that

$$
L_{p}(\breve{\mathbf{g}}, \breve{f})(1) \times L_{p}(K)\left(\psi^{-2} \mathbf{N}\right)=\mathfrak{f}(-1) \cdot L_{p}(f, K)\left(\psi^{-1} \mathbf{N}\right) .
$$

Since $L_{p}(K)\left((\psi)^{-2} \mathbf{N}_{K}\right)=L_{p}(K)\left(\psi^{-2}\right)$ (c.f. [17, p. 90-91]), Proposition 3.2 combined with Eqs. (29) and (12) shows that

$$
\int_{\breve{\gamma}} \breve{f} \cdot E_{1, \chi_{K, N}}=\lambda(\breve{f}, \breve{g}) \cdot \frac{\log _{E, p}^{2}\left(P_{\psi}\right)}{\log _{p}\left(u_{g}\right)}
$$

where

$$
\lambda(\breve{f}, \breve{g})=\mathcal{E} u l_{N}(-1) \cdot \mathfrak{f}_{\infty}(-1) \cdot \frac{\mathfrak{f}_{p}(f, \psi)}{\mathfrak{f}_{p}(\psi)} .
$$

From Eq. (39), we hence derive that

$$
\mathfrak{f}_{\infty}(-1)=-\frac{\Im\left(D_{K} c^{2}\right)}{\Im(N)} \cdot \frac{N}{D_{K} c^{2} \cdot h_{c}} \cdot 2^{-\sharp q \mid\left(D_{K}, N_{E}\right)} \cdot \psi(\mathfrak{N}) \in \mathbb{Q}_{\psi}\left(f_{N}\right)^{\times} .
$$

By Lemma $4.2, \lambda(\breve{f}, \breve{g}) \in \mathbb{Q}_{\psi}\left(f_{N}\right)^{\times}$hence the first statement of Theorem 1.4 follows. For the second part of our main theorem, assume that $N=D_{K}=N_{E}$ and $c=1$, and put $\breve{f}=f, \breve{g}=g$, and $\breve{h}=h=E_{1, \chi_{K}}$. In this setting, $\mathcal{E} u l_{N}(-1)=1$ and

$$
\lambda(f, g)=\mathfrak{f}_{\infty}(-1) \cdot \frac{\mathfrak{f}_{p}(f, \psi)}{\mathfrak{f}_{p}(\psi)} .
$$

Moreover, it follows from genus theory that $g_{K}:=\left[\mathrm{Cl}_{K}: \mathrm{Cl}_{K}^{2}\right]=2^{\# q \mid D_{K}-1}$ so that $\mathfrak{f}_{\infty}(-1)=\frac{-1}{2 h_{K} g_{K}}$. The proof follows after combining that with Formulae (12) and (29). 


\section{References}

1. Bertolini, M., Darmon, H.: Kato's Euler system and rational points on elliptic curves I: a p-adic Beilinson formula. Isr. J. Math. 199(1), 163-188 (2014)

2. Bellaïche, J., Dimitrov, M.: On the eigencurve at classical weight one points. Duke Math. J. http:// math.univ-lille1.fr/mladen/

3. Bertolini, M., Darmon, H., Prasanna, K.: Generalised Heegner cycles and $p$-adic Rankin L-series. Duke Math J. 162(6), 1033-1148 (2013)

4. Bertolini, M., Darmon, H., Prasanna, K.: p-Adic Rankin L-series and rational points on CM elliptic curves. Pac. J. Math. 260(2), 261-303 (2012)

5. Bertolini, M., Darmon, H., Rotger, V.: Beilinson-Flach elements and Euler systems I: syntomic regulators and p-adic Rankin L-series. J. Algebraic Geom. 24, 355-378 (2015)

6. Bertolini, M., Darmon, H., Rotger, V.: Beilinson-Flach elements and Euler systems II: the Birch and Swinnerton-Dyer conjecture for Hasse-Weil-Artin L-series. J. Algebraic Geom. 24, 569-604 (2015)

7. Cox, D.A.: Primes of the Form $x^{2}+n y^{2}$ : Fermat, Class Field Theory and Complex Multiplication. Wiley, New York (1989)

8. Darmon, H.: Rational points on modular elliptic curves. In: CBMS Regional Conference Series in Mathematics, 101. Published for the Conference Board of the Mathematical Sciences, Washington, DC; by the American Mathematical Society, Providence (2004)

9. Darmon, H., Dasgupta, S.: Elliptic units for real quadratic fields. Ann. Math. 163(1), 301-346 (2006)

10. Darmon, H., Lauder, A., Rotger, V.: Stark points and p-adic iterated integrals attached to modular forms of weight one. Forum Math. Pi 3, e8-95 (2015)

11. Darmon, H., Pollack, R.: The efficient calculation of Stark-Heegner points via overconvergent modular symbols. Isr. J. Math. 153, 319-354 (2006)

12. Darmon, H., Rotger, V.: Diagonal cycles and Euler systems I: a p-adic Gross-Zagier formula. Ann. Scie. de 1'Ecol. Norm. Supér. 47(4), 779-832 (2014)

13. Darmon, H., Rotger, V.: Diagonal cycles and Euler systems II: $p$-adic families and the Birch and Swinnerton-Dyer conjecture. J. Am. Math. Soc. (to appear)

14. De Shalit, E.: Iwasawa Theory of Elliptic Curves with Complex Multiplication. $p$-Adic $L$-Functions. Academic Press, Boston (1987)

15. Dokchitser, V.: $L$-functions of non-abelian twists of elliptic curves. Ph.D. dissertation, Cambridge (2005)

16. Gross, B.: Heegner points on $X_{0}(N)$, Modular forms (Durham,1983): Ellis Horwood Ser. Math. Appli.: Statist. Oper. Res. Horwood, Chichester, pp. 87-105 (1984)

17. Gross, B.: On the factorization of $p$-adic L-series. Invent. Math. 57(1), 83-95 (1980)

18. Gross, B., Zagier, D.: Heegner points and derivatives of $L$-series. Invent. Math. 84, 225-320 (1986)

19. Hida, H.: Congruences of cusp forms and special values of their zeta functions. Invent. Math. 63, 225-261 (1981)

20. Hida, H.: Elementary Theory of $L$-Functions and Eisenstein Series, vol. 26. London Mathematical Society Student Texts, Cambridge (1993)

21. Kani, E.: The space of binary theta series. Ann. Sci. Math. Quebec 36, 501-534 (2012)

22. Kani, E.: Binary theta series and modular forms with complex multiplication. Int. J. Number Theory 10, 1025-1042 (2014)

23. Katz, N.M.: p-Adic interpolation of real analytic Eisenstein series. Ann. Math. 104(3), 459-571 (1976)

24. Katz, N.M.: p-Adic L-functions for CM fields. Invent. Math. 49, 199-297 (1978)

25. Khare, C., Wintenberger, J.-P.: Serre's modularity conjecture (I). Invent. Math. 178, 485-504 (2009)

26. Lauder, A.: Computations with classical and p-adic modular forms. LMS J. Comput. Math. 14, 214-231 (2011)

27. Lauder, A.: Efficient computation of Rankin $p$-adic $L$-functions. In: Boeckle G., Wiese G. (eds.) Computations with Modular Forms, Proceedings of a Summer School and Conference, Heidelberg, August/September 2011, Springer, Berlin, pp. 181-200 (2014)

28. Mazur, B., Tate, J., Teitelbaum, J.: On $p$-adic analogues of the conjectures of Birch and SwinnertonDyer. Invent. Math. 84(1), 1-48 (1986)

29. Petersson, H.: Uber die Berechnung der skalarprodukte ganzer modulformen. Commun. Math. Helv. 22, 168-199 (1949)

30. Prasad, D.: Trilinear forms for representations of GL(2) and local epsilon factors. Compos. Math. 75, $1-46$ (1990) 
31. Roberts, G.: Unites elliptiques et formules pour le nombre de classes des extensions abeliennes d'un corps quadratique imaginaire. Bull. Soc. Math. France, Mém. 36, 77 (1973)

32. Rohrlich, D.: Root Numbers, Notes from PCMI/IAS: Arithmetic of L-Functions at Park City Mathematics Institute, Park City (2009)

33. Rubin, K.: $p$-Adic $L$-functions and rational points on elliptic curves with complex multiplication. Invent. Math. 107(2), 323-350 (1992)

34. Rubin, K.: p-Adic variants of the Birch and Swinnerton-Dyer conjecture for elliptic curves with complex multiplication. In: $p$-Adic monodromy and the Birch and Swinnerton-Dyer conjecture (Boston, MA, 1991), pp. 71-80, Contemp. Math., vol. 165, Am. Math. Soc., Providence (1994)

35. Shimura, G.: The special values of the zeta functions associated with cusp forms. Commun. Pure. Appl. Math. 29, 783-804 (1976)

36. Silverman, J.H.: Advanced topics in the arithmetic of elliptic curves. Grad. Texts Math., vol. 151 (1994) 